\title{
Analytical Approximate Expression for Cocurrent Imbibition during Immiscible Two-Phase Flow through Porous Media
}

\author{
Saroj R. Yadav and Manoj N. Mehta \\ Applied Mathematics and Humanities Department, S. V. National Institute of Technology, Surat 395007, India \\ Correspondence should be addressed to Manoj N. Mehta; mnm@ashd.svnit.ac.in
}

Received 19 July 2013; Revised 4 December 2013; Accepted 12 December 2013; Published 29 January 2014

Academic Editor: Chaudry M. Khalique

Copyright ( 2014 S. R. Yadav and M. N. Mehta. This is an open access article distributed under the Creative Commons Attribution License, which permits unrestricted use, distribution, and reproduction in any medium, provided the original work is properly cited.

\begin{abstract}
Cocurrent and countercurrent imbibitions are the crucial mechanism in many multiphase flow processes. In cocurrent imbibition wetting phase displaces nonwetting phase such that the nonwetting phase moves in the same direction to the wetting phase, whereas in countercurrent imbibitions wetting and non-wetting phase flow in opposite directions. However for cocurrent imbibitions, mathematical models need total flux condition as both phases flow in the same direction. Thus cocurrent imbibitions have been considered neglecting pressure gradient of nonwetting phase and only pressure gradient of displacing phase is considered which gives additional velocity to the displacing phase. An approximate analytical solution is derived by the method of small parameter; an approximate expression for the wetting phase saturation has been obtained. From analytical expression graphical presentation of saturation of wetting phase shows that cocurrent imbibition is faster than countercurrent imbibition. Also, the small parameter is chosen from initial wetting phase saturation and wetting phase saturation at imbibition phase, thus giving comparative behavior of imbibition at initial and later stage. It is shown that cocurrent imbibition proceeds faster with more amount of wetting phase present in porous matrix.
\end{abstract}

\section{Introduction}

Imbibition is one of the most important mechanisms, if wetting phase (like water and brine) enters in porous matrix and displaces nonwetting phase (like air, oil, and nonaqueous phase liquids (NAPL)). Imbibition is defined as the displacement of nonwetting phase (generally air or oil) by wetting phase (generally water), where driving force is capillary pressure. During imbibition two main types of flow modes are recognized: cocurrent flow in which displacing (wetting) phase and displaced (nonwetting) phase flow in the same direction and countercurrent flow in which displacing phase flows in the opposite direction to displaced phase. Imbibition in water-wet porous media is commonly considered to be counter current, but studies have shown that when a porous matrix block is partially covered by wetting fluid, flow is dominated by cocurrent imbibition, not countercurrent [1]. Such situation may arise during water flowing in unsaturated soil or oil recovery from fractured porous matrix. Though permeability of fracture is higher having relatively low volume, porous matrix with low permeability and higher volume contains major volume of oil. During water flooding process, water quickly surrounds oil-saturated (water wet) porous matrix. Imbibition may occur as cocurrent or counter current, depending on fracture network and water injection rate. Having an oil filled porous matrix exposed from both sides to water, imbibition is countercurrent from each end. If the matrix is exposed so that one end is in the water and the other is in the oil, then imbibition is cocurrent with water entering from one end and oil leaving from the other end (Figure 1).

In spite of the fact that cocurrent imbibition is faster and more efficient countercurrent imbibitions have received considerable attention in the literature; comparatively less studies are undertaken to understand cocurrent imbibitions experimentally as well as theoretically. Through a detailed study of the governing equations and boundary conditions, significant insight has been provided into the physical differences between co- and countercurrent imbibitions by some authors [1-3]. Experimental studies have shown that there are significant recovery differences between cocurrent and countercurrent imbibition [4-6]. Pooladi-Darvish and Firoozabadi [1] developed a numerical model and studied the similarities and 


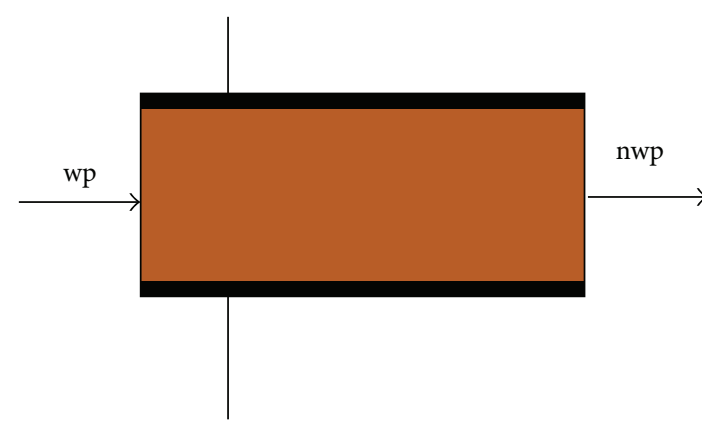

(a)

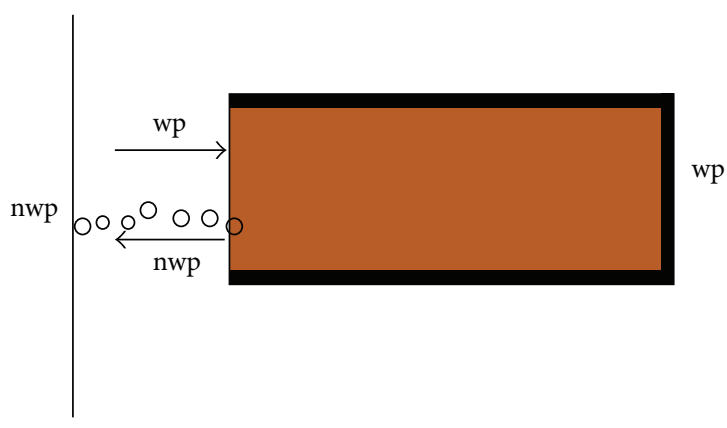

(b)

FIGURE 1: Cocurrent and countercurrent imbibition when nonwetting phase is displaced by wetting phase.

differences of cocurrent and countercurrent imbibition and point out the consequences for practical applications.

In the present study cocurrent imbibition is formulated neglecting the nonwetting phase pressure gradient and considering that capillary pressure gradient and wetting fluid pressure gradient are equal $[1,3])$. Darcy-like formulation of the flow equation is assumed to be sufficient for analysis, in which the local wetting phase (water) saturation $S_{w}(x, t)$ obeys a differential equation of the diffusion type with one additional convective term. Wetting and nonwetting phases flow in the same direction; thus the sum of Darcy's velocities is taken as nonzero. Also specific results for the dependence of relative permeability and capillary pressure, on phase saturation, have been taken from standard literature. Governing differential equation is formulated and solved by method of small parameter; an approximate expression for saturation of wetting phase $S_{w}(x, t)$ has been obtained.

\section{Governing Flow Equation for Imbibition}

The one-dimensional horizontal flow of two immiscible flowing phases can be described by the multiphase extension of the Darcy law for each phase, which describes the seepage velocity of each phase because of a gradient in the phase pressures:

$$
\begin{aligned}
& v_{w}=-\frac{k_{r w}}{\mu_{w}} k\left(\frac{\partial P_{w}}{\partial x}\right), \\
& v_{n}=-\frac{k_{r n}}{\mu_{n}} k\left(\frac{\partial P_{n}}{\partial x}\right),
\end{aligned}
$$

where $v_{w}, v_{n}$ are the velocity of the wetting and the nonwetting phases, respectively, $k$ is the absolute permeability, and $\mu_{w}, \mu_{n}$ are the viscosity of the wetting and the nonwetting phases, respectively. Also $k_{r w}=k_{r w}\left(S_{w}\right), k_{r n}=k_{r n}\left(S_{w}\right)$ are the relative permeability of the wetting and the nonwetting phase, respectively, which are function of the wetting phase saturation and describe the impairment of one fluid phase by the other.

The definition of capillary pressure $P_{c}$ as the pressure discontinuity between the flowing phases yields $P_{c}=P_{n}-P_{w}$ [7]. Hence, pressure gradients of both phases are related to the gradient of the capillary pressure, which was first introduced for two-phase flow in porous media by Leveratt [8]; that is,

$$
\begin{gathered}
\frac{\partial P_{c}}{\partial x}=\frac{\partial P_{n}}{\partial x}-\frac{\partial P_{w}}{\partial x} . \\
\text { Total velocity } v_{t}=v_{w}+v_{n},
\end{gathered}
$$

is the sum of the velocities of wetting and nonwetting phases. Using total velocity and from (1) and (2) velocity of wetting phase can be written as

$$
v_{w}=\frac{v_{t}}{\left(1+k_{r n} \mu_{w} / k_{r w} \mu_{n}\right)}+\frac{k\left(k_{r n} / \mu_{n}\right)\left(\partial P_{c} / \partial x\right)}{\left(1+k_{r n} \mu_{w} / k_{r w} \mu_{n}\right)} .
$$

Equation (4) gives the velocity of wetting phase as the sum of two terms; first term on right is dictated by the rate at which fluid is entering at the boundary. The second term occurs due to additional impelling force resulting from gradient of capillary pressure (effect of wettability).

Neglecting phase density variation, the equation of continuity for wetting phase may be written as

$$
\phi \frac{\partial S_{w}}{\partial t}+\frac{\partial v_{w}}{\partial x}=0
$$

where $\phi \in(0,1)$ is porosity of porous matrix.

Considering capillary pressure as function of phase saturation of displacing fluid [1] in (5) and using (4) for velocity of displacing phase give

$$
\left(\frac{\partial S_{w}}{\partial t}\right)=-\frac{\partial}{\partial x}\left(\frac{v_{t}}{\phi} f\left(S_{w}\right)+D_{c}\left(S_{w}\right) \frac{\partial S_{w}}{\partial x}\right),
$$

where $D_{c}\left(S_{w}\right)=(k / \phi)\left(f\left(S_{w}\right)\left(k_{r n} / \mu_{n}\right)\right)\left(d P_{c} / d S_{w}\right)$ is known as capillary diffusion coefficient and function $f\left(S_{w}\right)$ is given by the following expression:

$$
f\left(S_{w}\right)=\frac{1}{1+\left(k_{r n} / k_{r w}\right)\left(\mu_{n} / \mu_{w}\right)} .
$$

The above partial differential equation has been previously derived by several authors which describes one-dimensional, immiscible, incompressible, isothermal, two-phase flow through homogeneous, horizontal porous media. Imbibition 
is cocurrent or countercurrent, that is, described by the second term containing $v_{t}$, for countercurrent flow $v_{t}=0$ and for cocurrent flow $v_{t} \neq 0$. Equation (6) has been formulated earlier for nonlinear relation between capillary pressure and phase saturation of displacing fluid so as to describe imbibition model by McWhorter and Sunada [2], PooladiDarvish and Firoozabadi [1], and Le Guen and Kovscek [3]. For countercurrent and cocurrent imbibition, the coefficient of diffusion $D_{c}\left(S_{w}\right)$ is function of saturation of wetting phase only because capillary pressure and relative permeability of wetting phase depend only on wetting fluid saturation. In (6) $v_{t}$ is unknown and thus an additional equation, that is, the pressure equation with initial and boundary conditions is required to complete the formulation. Only a few analytical solutions of this equation are known because of strong nonlinearity due to capillary drive in $D_{c}$ and $f$. Thus, generally two approaches are taken to solve this equation. In first approach, closed form solutions are determined restricting the $k_{r w}\left(S_{w}\right), k_{r n}\left(S_{w}\right)$, and $P_{c}\left(S_{w}\right)$ to particular nonlinearities; otherwise more general nonlinearities are chosen and the resulting exact analytical expression is mostly nonlinear expression that generally needs to be solved numerically [9]. In the first approach, the specific form of the nonlinearities is considered. In the present paper first approach has been taken to find approximate analytical solution.

Some studies of the imbibition process have assumed that the pressure gradient in the displaced oil phase may be neglected [3]. This assumption is based on the common practice in hydrology, where the mathematical formulation of unsaturated water flow ignores the air pressure gradient [10]. Under this assumption, (2) gives

$$
\frac{\partial P_{c}}{\partial x}=-\frac{\partial P_{w}}{\partial x} .
$$

From (8) simplification of (6) results in

$$
\frac{\partial S_{w}}{\partial t}=-\frac{\partial}{\partial x}\left\{D\left(S_{w}\right) \frac{\partial S_{w}}{\partial x}\right\}
$$

where $D\left(S_{w}\right)=\left(k k_{r w} / \phi \mu_{w}\right)\left(d P_{c} / d S_{w}\right)$ is diffusion coefficient.

Following Scheidegger and Johnson [11], relation between relative permeability of wetting and nonwetting phases and saturation of wetting phase can be written as

$$
\begin{gathered}
k_{r w}=S_{w}, \\
k_{r n}=1-\alpha S_{w}, \quad \alpha=1.11 .
\end{gathered}
$$

Babchin and Nasr [12] suggested that when both the phases are continuous then the capillary pressure gradient (in present notations) can be written as $\Delta P_{c}=\left(\gamma_{n s}-\gamma_{w s}\right) S_{v} \Delta S_{n}$, where $S_{v}$ is the specific surface area of homogeneous porous media and $\gamma_{n s}$ and $\gamma_{w s}$ are native fluid-solid and displacing fluid-solid specific surface energies, respectively. Also Mehta [13] suggested the presence of a linear relation between capillary pressure and phase saturation of displacing phase when external force does not apply. Hence, capillary pressure and wetting phase saturation can be considered related by expression $P_{c}=-\beta S_{w}$, where $\beta$ is constant of proportionality
[14]. Thus substitution of capillary pressure and wetting phase relation and expression of relative permeability of wetting phase from (10) in (9) gives

$$
\frac{\partial S_{w}}{\partial t}=-\frac{\partial}{\partial x}\left\{\frac{(-\beta) k S_{w}}{\phi \mu_{w}} \frac{\partial S_{w}}{\partial x}\right\} .
$$

Equation (11) is the desired nonlinear partial differential equation in wetting phase saturation, which describes the linear cocurrent imbibition. The governing differential equation of counter current imbibition is given in Mehta and Yadav [15] which differs in diffusivity co-efficient.

Set of suitable boundary and initial conditions associated with (11) are

$$
\begin{aligned}
& S_{w}(0, t)=S_{1} \quad \text { at } x=0, t \geq 0, \\
& S_{w}(x, 0)=S_{2} \quad \text { at } t=0, x>0,
\end{aligned}
$$

where $S_{1}$ is the saturation of wetting phase at the imbibition phase, as only displacing phase, that is, wetting phase, flows through imbibition face $0<S_{1}=1-S_{r n}<1$ at $x=0$ for $t>0$, where $S_{r n}$ is irreducible saturation of nonwetting phase. $S_{2}$ is the initial saturation of the wetting phase in the porous matrix under consideration, at $t=0$ for $x>0$.

Equation (11) along with initial and boundary conditions (12a) and (12b) is a nonlinear differential equation which describes the cocurrent imbibition phenomenon in a homogeneous porous cylindrical matrix with impervious cylindrical bounding surfaces. With all the linear relations used to derive (11), it now becomes possible to use some of the calculations that occur in the theory of motion with free surface.

\section{Solution of the Problem}

Introducing the dimensionless variables:

$$
X=\frac{x}{L}, \quad T=\frac{\beta k t}{\phi L^{2} \mu_{w}},
$$

where $0 \leq X \leq 1$ and $0 \leq T \leq 1$ in (11), it reduces to

$$
\frac{\partial S_{w}}{\partial T}=\frac{\partial}{\partial X}\left(S_{w} \frac{\partial S_{w}}{\partial X}\right) .
$$

From (12a) and (12b), initial and boundary conditions are

$$
\begin{aligned}
& S_{w}(0, T)=S_{1}, \quad \text { at } X=0, T \geq 0, \\
& S_{w}(X, 0)=S_{2}, \quad \text { at } T=0, X>0 .
\end{aligned}
$$

Introducing the transformations as below;

$$
S_{w}(X, T)=S_{1} \xi(\eta), \quad \eta=\frac{X}{2 \sqrt{S_{1} T}}
$$

in (14) results in

$$
\frac{d^{2} \xi^{2}}{d \eta^{2}}+4 \eta \frac{d \xi}{d \eta}=0
$$


Initial and boundary conditions given in (15a) and (15b) will transform to

$$
\begin{gathered}
\xi(0)=1, \\
\lim _{\eta \rightarrow \infty} \xi(\eta)=\frac{S_{2}}{S_{1}} .
\end{gathered}
$$

We now seek an approximate analytical solution for (17a) through an exposed method by power series expansion in powers of a small parameter [16]. Exposed method can be applied to (17a) with conditions (17b) and (17c), as conditions are considered to be constant. Assuming that solution of (17a) can be expressed in the form of a power series in powers of small parameter $\varepsilon$ gives

$$
\xi(\eta)=1+\varepsilon \xi_{*}+\varepsilon^{2} \xi_{* *}+\varepsilon^{3} \xi_{* * *}+\cdots
$$

where $\xi_{*}(\eta), \xi_{* *}(\eta), \xi_{* * *}(\eta), \ldots$ are some functions of $\eta$. In order to satisfy initial and boundary conditions in (17b) and $(17 \mathrm{c}), \xi_{*}(\eta), \xi_{* *}(\eta), \xi_{* * *}(\eta), \ldots$ are subject to the following conditions:

$$
\begin{gathered}
\xi_{*}(0)=\xi_{* *}(0)=\xi_{* * *}(0)=\cdots=0, \\
\xi_{* *}(\infty)=\xi_{* * *}(\infty)=\cdots=0 .
\end{gathered}
$$

In order to satisfy condition (17b) at infinity, we must set $\lim _{\eta \rightarrow \infty} \xi(\eta)=S_{2} / S_{1}=1+\varepsilon \xi_{*}(\infty)$, choosing $\xi_{*}(\infty)$ such that $\lim _{\eta \rightarrow \infty} \xi_{*}(\eta)=1$.

Thus the value for the small parameter $\varepsilon$ can be obtained as follows:

$$
\varepsilon=\frac{S_{2}-S_{1}}{S_{1}} .
$$

Substituting the series (18) in (17a) and equating like powers of $\varepsilon$ gives

$$
\begin{gathered}
\frac{d^{2} \xi_{*}}{d \eta^{2}}+2 \eta \frac{d \xi_{*}}{d \eta}=0, \\
\frac{d^{2} \xi_{* *}}{d \eta^{2}}+2 \eta \frac{d \xi_{* *}}{d \eta}-\eta \xi_{*} \frac{d \xi_{*}}{d \eta}=0, \\
\frac{d^{2} \xi_{* * *}}{d \eta^{2}}+2 \eta \frac{d \xi_{* * *}}{d \eta}+\frac{3}{4} \eta \xi_{*}^{2} \frac{d \xi_{*}}{d \eta} \\
-\eta\left\{\xi_{*} \frac{d \xi_{* *}}{d \eta}+\xi_{* *} \frac{d \xi_{*}}{d \eta}\right\}=0,
\end{gathered}
$$

The first approximation in (21) together with conditions in (19) gives probability function [16]

$$
\xi_{*}(\eta)=\frac{2}{\sqrt{\pi}} \int_{0}^{\eta} e^{-\tau^{2}} d \tau .
$$

Integrating by parts after simplification of the second equation in (21) and using the conditions in (19), the expression of second approximation can be given as follows:

$$
\begin{aligned}
\xi_{* *}(\eta)= & \frac{1}{\pi}\left(1-e^{-2 \eta^{2}}\right)-\frac{1}{\sqrt{\pi}} \eta e^{-2 \eta^{2}} \xi_{*}-\frac{1}{2}\left(\xi_{*}\right)^{3} \\
& +\left(\frac{1}{2}-\frac{1}{\pi}\right) \xi_{*} .
\end{aligned}
$$

Similarly, for the third approximation the expression is obtained as follows:

$$
\begin{aligned}
\xi_{* * *}(\eta)= & \frac{1}{2} \xi_{*}^{3}+\frac{9}{4 \sqrt{\pi}} \eta e^{-\eta^{2}} \xi_{*}^{2}-\frac{1}{2 \sqrt{\pi}} \eta^{3} e^{-\eta^{2}} \xi_{*}^{2} \\
& +\frac{3}{\pi} e^{-\eta^{2}} \xi_{*}-\frac{1}{\pi} \eta^{2} e^{-2 \eta^{2}} \xi_{*}-\frac{\eta e^{-\eta^{2}}}{\pi \sqrt{\pi}} \\
& -\frac{\eta e^{-3 \eta^{2}}}{2 \pi \sqrt{\pi}}-\frac{3 \sqrt{3}}{4 \pi} \xi_{*}(\eta \sqrt{3})+\left(1-\frac{2}{\pi}\right) \xi_{* *} \\
& +\left(\frac{3 \sqrt{3}}{4 \pi}-\frac{1}{2}\right) \xi_{*} .
\end{aligned}
$$

From (22), (23), and (24), considering $\xi_{*}(\eta), \xi_{* *}(\eta)$, $\xi_{* * *}(\eta), \ldots$ in series (18) and using (16), the solution of (14) can be given as follows,

$$
S_{w}(X, T)=S_{1} \xi(\eta)=S_{1}\left(1+\varepsilon \xi_{*}+\varepsilon^{2} \xi_{* *}+\varepsilon^{3} \xi_{* * *}+\cdots\right),
$$

where value of parameter $\varepsilon$ is as in (20) with conditions in (19). Thus (25) gives saturation of wetting phase at any time $T$ and at distance $X$, where $T$ and $X$ are dimensionless time and distance, respectively.

\section{Discussion and Conclusions}

An approximate analytical solution in infinite series is obtained for cocurrent imbibition, which satisfies initial and boundary conditions with terms containing negative exponential term in coefficients. From the expressions given in (22), (23), and (24) the coefficient of the series may be considered [17] as shown in Table 1. Also, for the free surface Polubarinova-Kochina concluded from Table 1 that the series up to third-degree approximation was not valid for the extreme cases (zero initial and boundary conditions) considered. But for the cocurrent imbibition occurring due to water injection in secondary oil recovery this method works. As discussed earlier, $0<S_{1}=1-S_{r n}<1$ at $x=0$ for $t>0$, where $S_{r n}$ is irreducible saturation of nonwetting phase and the initial (irreducible) saturation of the wetting phase in the porous matrix under consideration is $0<S_{2}$ at $t=0$ for $x>0$. Also saturation of wetting phase is relatively more due to spontaneous imbibition during primary recovery. One more advantage of this method lies in using only one boundary condition, that is, $x=0$. Also the saturation of wetting phase increases at $x=L$ gradually hence may be left as free boundary. From the expressions (22), (23), and (24) and from Table 1, for the coefficients of the series observation can be made that for $\eta \rightarrow \infty$ 
TABLE 1

\begin{tabular}{|c|c|c|c|}
\hline$\eta$ & $\xi_{*}$ & $\xi_{* *}$ & $\xi_{* * *}$ \\
\hline 0 & 0 & 0 & 0 \\
\hline 0.1 & 0.1125 & 0.0141 & -0.0039 \\
\hline 0.2 & 0.2227 & 0.0160 & -0.0081 \\
\hline 0.3 & 0.3286 & 0.0073 & -0.0090 \\
\hline 0.4 & 0.4284 & -0.0092 & -0.0049 \\
\hline 0.5 & 0.5205 & -0.0300 & 0.0039 \\
\hline 0.6 & 0.6039 & -0.0519 & 0.0159 \\
\hline 0.7 & 0.6778 & -0.0718 & 0.0280 \\
\hline 0.8 & 0.7421 & -0.0874 & 0.0373 \\
\hline 0.9 & 0.7969 & -0.0975 & 0.0422 \\
\hline 1 & 0.8427 & -0.1017 & 0.0418 \\
\hline 1.1 & 0.8802 & -0.1004 & 0.0368 \\
\hline 1.2 & 0.9103 & -0.0946 & 0.0281 \\
\hline 1.3 & 0.9340 & -0.0855 & 0.0194 \\
\hline 1.4 & 0.9523 & -0.0744 & 0.0078 \\
\hline 1.5 & 0.9661 & -0.0626 & -0.0011 \\
\hline 1.6 & 0.9764 & -0.0510 & -0.0079 \\
\hline 1.7 & 0.9838 & -0.0394 & -0.0125 \\
\hline 1.8 & 0.9891 & -0.0310 & -0.0147 \\
\hline 1.9 & 0.9928 & -0.0232 & -0.0151 \\
\hline 2 & 0.9953 & -0.0169 & -0.0141 \\
\hline 2.5 & 0.9996 & -0.0024 & -0.0047 \\
\hline 3 & 0.9999 & -0.0002 & -0.0006 \\
\hline 3.5 & 1 & -0.0 & -0.0001 \\
\hline 4 & 1 & -0.0 & -0.0001 \\
\hline
\end{tabular}

each coefficient approaches zero. The small parameter $\varepsilon$ has been chosen depending upon the ratio $\left(S_{2}-S_{1}\right) / S_{1}$, where $0<$ $S_{2}<S_{1}<1$. Hence the parameter $\varepsilon$ is small with negative sign which compensates the negative series coefficients occurring in Table 1; giving a convergent series in (25).

Saturation of displacing fluid $S_{w}$ increases at distance $x$ as time $t$ increases; graphical presentation of saturation profile in dimensionless variables is shown for cocurrent and countercurrent imbibition [15] with $S_{w}(X, 0)=S_{2}=0.2$. Saturation profile shows different behaviors during cocurrent and countercurrent imbibition.

Also, during cocurrent imbibition for given time $T$ saturation varies nonlinearly initially showing faster rate but slowly becomes linear after long time $T$ (Figure 2). Figure 3 shows saturation in porous matrix for countercurrent for the same initial and boundary condition. During countercurrent imbibition saturation profile shows the same behavior for all time $T$. From both saturation profiles it can be observed that saturation profile of wetting phase increases faster in cocurrent imbibition than in countercurrent imbibition.

Also, the expression (25) gives saturation of wetting phase through a power series expansion in powers of parameter $\varepsilon$. Thus for the purpose of comparison of cocurrent imbibition at different initial wetting phase saturation, saturation profile is depicted in Figure 4. These profiles show cocurrent imbibition is faster in later stages than primary or in other words connected wetting phase is dominant during the imbibition

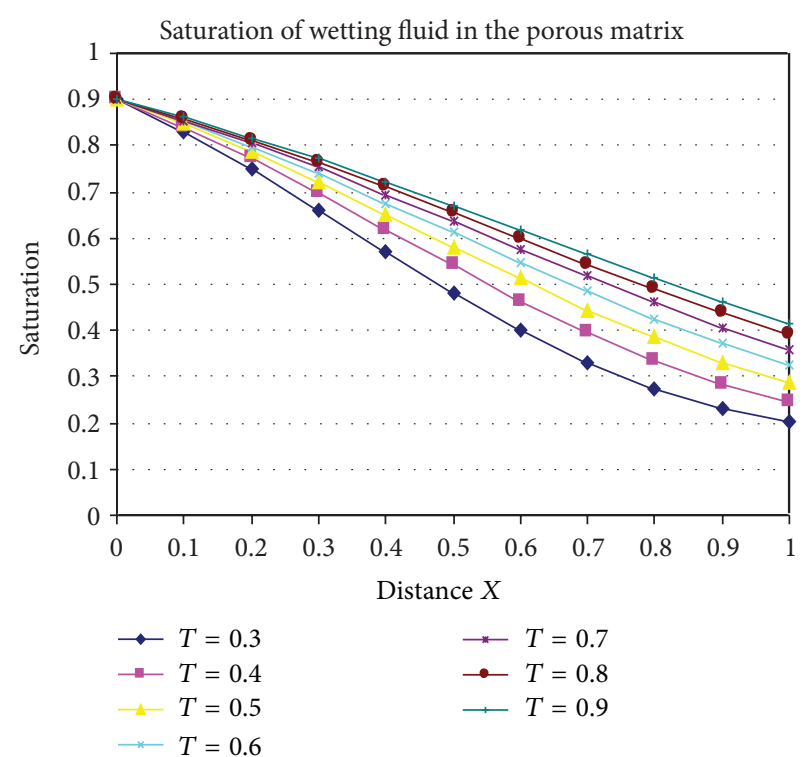

FIGURE 2: Saturation of wetting fluid versus distance during cocurrent imbibition.

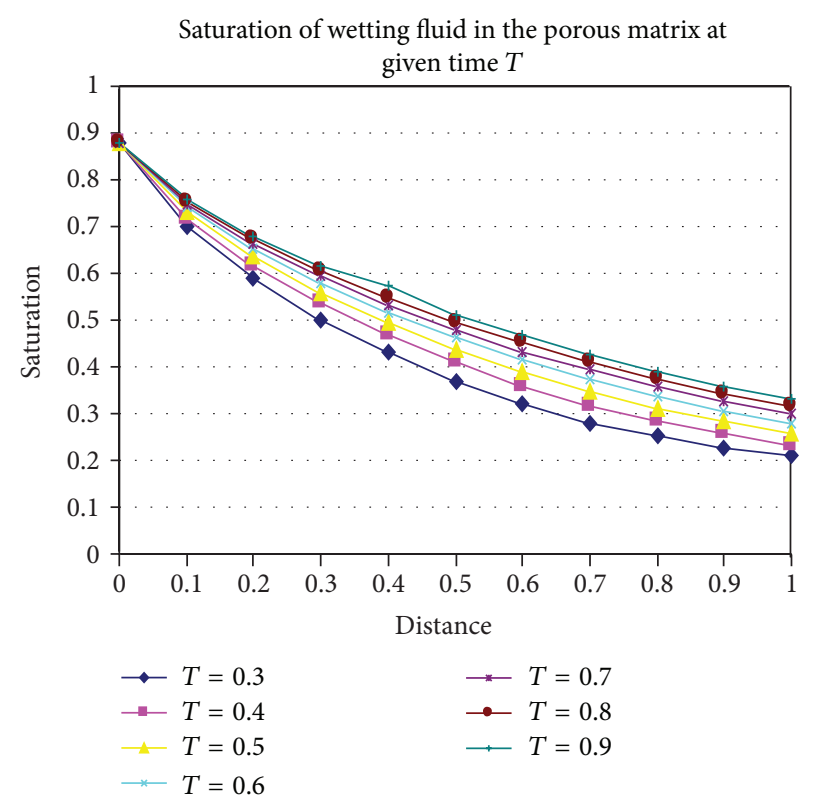

FIGURE 3: Saturation of wetting fluid versus distance during countercurrent imbibition.

mechanism. Earlier few authors $[18,19]$ argue over this aspect by conducting experimental work on countercurrent imbibition. They observed that secondary imbibition proceeds via the thickening of the preexisting wetting phase. The expression given by (25) shows faster progress in saturation profile as a consequence of the thickening of initially present wetting phase in the porous medium.

In summary, the present paper addresses cocurrent imbibition with some restrictive assumption on capillary pressure and relative permeability of wetting and nonwetting phases to derive approximate analytical solution in power series. The method may be applied to solve more general problems 


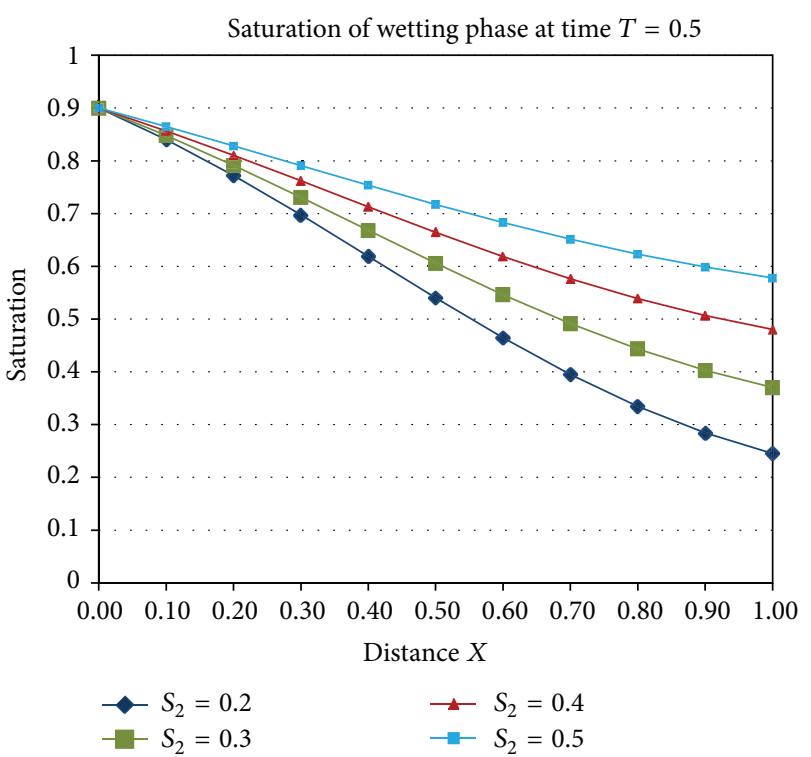

Figure 4: Saturation of wetting phase at fixed time for different initial conditions during cocurrent imbibition.

with nonlinear relation for capillary pressure and relative permeabilities, if resultant set of differential equations for series coefficients (as in (21)) is solvable. In conclusion we have found the approximate analytical expression for wetting phase saturation during cocurrent imbibition for a simplified model formulated by using linear relation curves. In spite of the restrictive formulation of the solution, saturation profile behavior shows agreement with available literature. The proposed method can be used to find solution for more general forms.

\section{Conflict of Interests}

The authors declare that there is no conflict of interests regarding the publication of this paper.

\section{Acknowledgments}

The authors thank the anonymous reviewers, whose comments have helped to greatly improve this paper.

\section{References}

[1] M. Pooladi-Darvish and A. Firoozabadi, "Cocurrent and countercurrent imbibition in a water-wet matrix block," SPE Journal, vol. 5, no. 1, pp. 3-11, 2000.

[2] D. B. McWhorter and D. K. Sunada, "Exact solutions for twophase flow," Water Resources Research, vol. 26, pp. 399-413, 1990.

[3] S. S. Le Guen and A. R. Kovscek, "Nonequilibrium effects during spontaneous imbibition," Transport in Porous Media, vol. 63, no. 1, pp. 127-146, 2006.

[4] B. Bourbiaux and F. Kalaydjian, "Experimental study of cocurrent and countercurrent flow in natural porous media," SPE Reservoir Engineering, vol. 5, no. 3, pp. 361-368, 1990.
[5] E. Unsal, G. Mason, N. R. Morrow, and D. W. Ruth, "Cocurrent and counter-current imbibition in independent tubes of non-axisymmetric geometry," Journal of Colloid and Interface Science, vol. 306, no. 1, pp. 105-117, 2007.

[6] E. Unsal, G. Mason, D. W. Ruth, and N. R. Morrow, "Coand counter-current spontaneous imbibition into groups of capillary tubes with lateral connections permitting cross-flow," Journal of Colloid and Interface Science, vol. 315, no. 1, pp. 200209, 2007.

[7] A. E. Scheidegger, The Physics of Flow through Porous Media, The Macmillan, New York, NY, USA, 1960.

[8] M. C. Leveratt, "Capillary behaviour in porous solids," Transactions of the AIME, vol. 142, pp. 152-169, 1941.

[9] K. S. Schmid, S. Geiger, and K. S. Sorbie, "Semianalytical solutions for concurrent and countercurrent imbibition and dispersion of solutes in immiscible two-phase flow," Water Resources Research, vol. 47, Article ID W02550, 2011.

[10] H. J. Morel-Seytoux, "Two-Phase Flow in Porous Media," in Advances in Hydroscience, R. J. M. Dewiest, Ed., Academic Press, San Diego, Calif, USA, 9th edition, 1973.

[11] A. E. Scheidegger and E. F. Johnson, "The statistical behavior of instabilities in displacement processes in porous media," Canadian Journal of Physics, vol. 39, pp. 326-334, 1961.

[12] A. J. Babchin and T. N. Nasr, "Analytical model for the capillary pressure gradient in oil-water-rock system," Transport in Porous Media, vol. 65, no. 2, pp. 359-362, 2006.

[13] M. N. Mehta, Asymptotic expansion of fluid flow through porous media [Ph.D. thesis], South Gujarat University, Surat, India, 1977.

[14] K. R. Patel, M. N. Mehta, and T. R. Patel, "A mathematical model of imbibition phenomenon in heterogeneous porous media during secondary oil recovery process," Applied Mathematical Modelling, vol. 37, no. 5, pp. 2933-2942, 2013.

[15] M. N. Mehta and S. R. Yadav, "Analytical Approximate expression for Primary Imbibition front in Homogeneous Porous Media," International Journal of Mathematical Sciences and Engineering Applications, vol. 2, no. 2, pp. 155-162, 2008.

[16] P. Ya. Polubarinova-Kochina, Theory of Ground Water Movement, Princeton University Press, Princeton, NJ, USA, 1962.

[17] P. Y. Polubarinova-Kochina, "On a non-linear partial differential equation, occurring in seepage theory," Doklady Akademii Nauk, vol. 36, no. 6, 1948.

[18] B. A. Baldwin and E. A. Spinler, "In-situ saturation development during spontaneous imbibition," in Proceedings of the International Society of Core Analysis, SCA-9922, pp. 1-11, 1999.

[19] Y. Meleán, D. Broseta, and R. Blossey, "Imbibition fronts in porous media: effects of initial wetting fluid saturation and flow rate," Journal of Petroleum Science and Engineering, vol. 39, no. 3-4, pp. 327-336, 2003. 


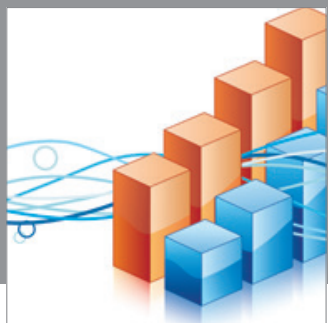

Advances in

Operations Research

mansans

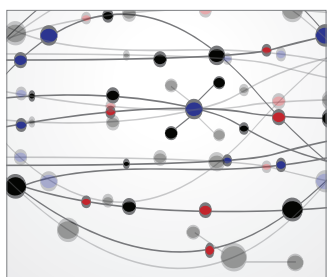

The Scientific World Journal
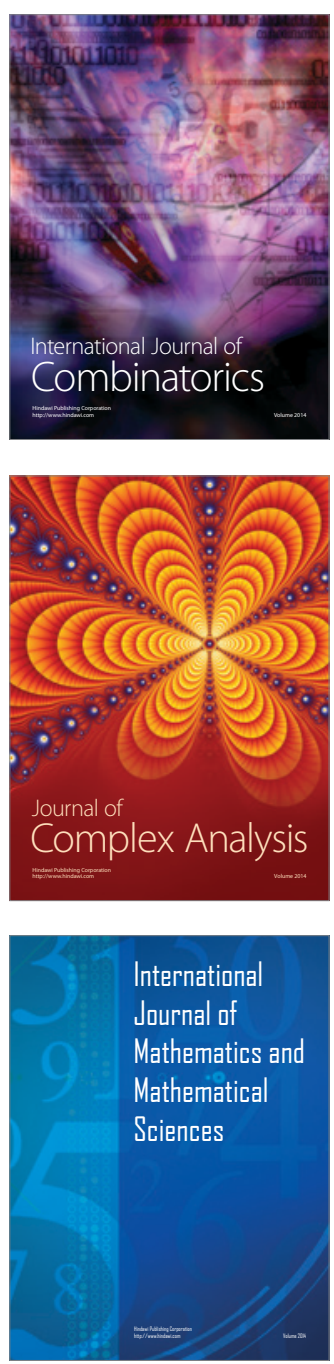
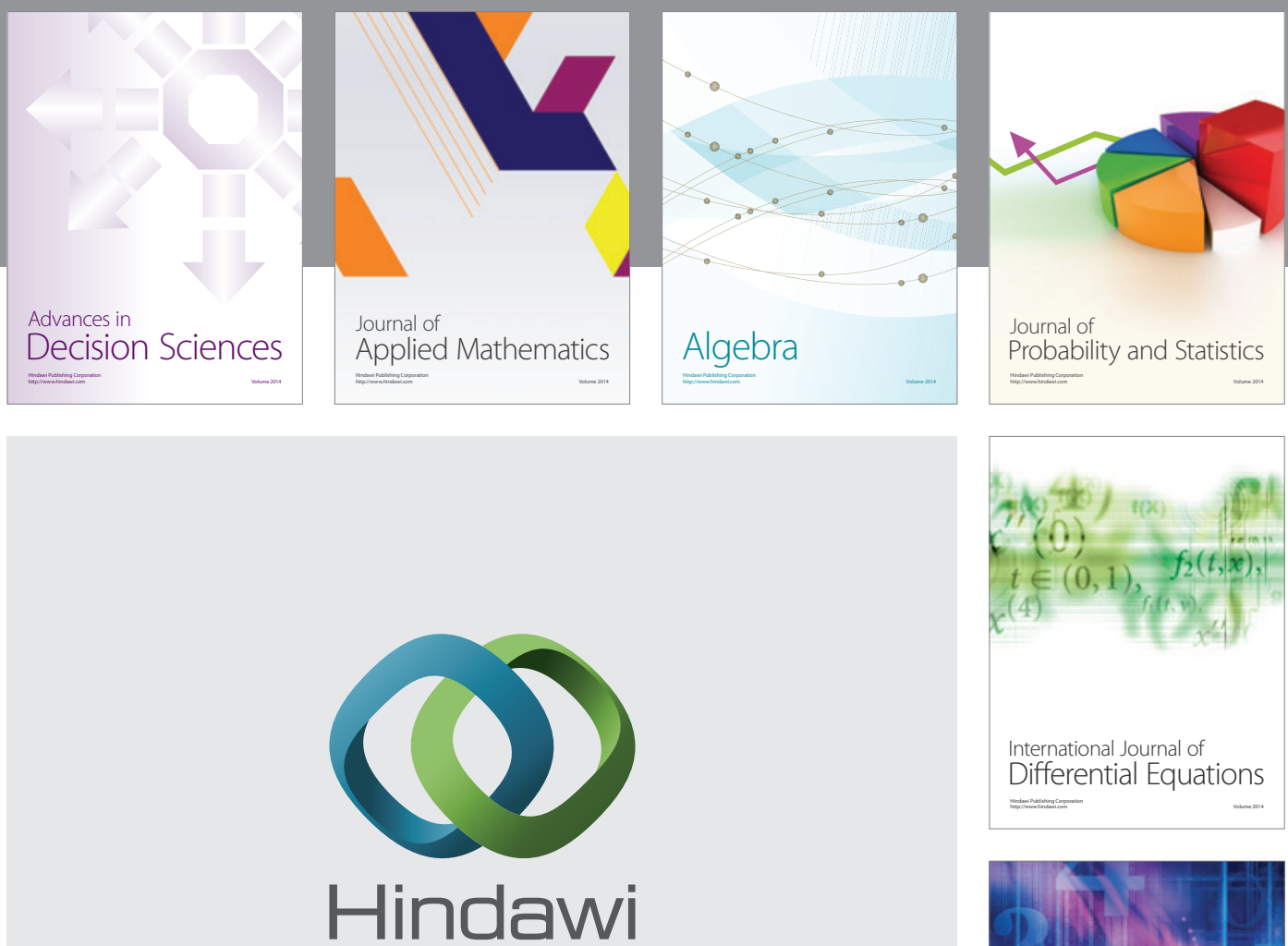

Submit your manuscripts at http://www.hindawi.com
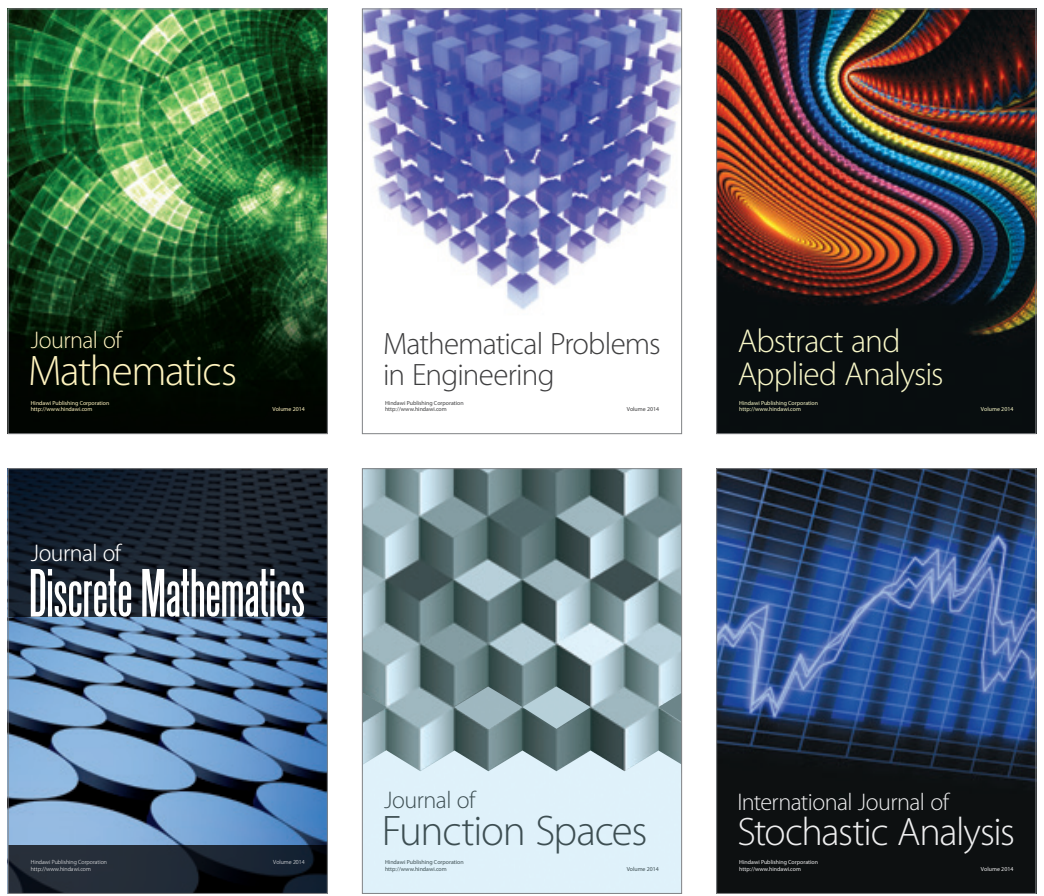

Journal of

Function Spaces

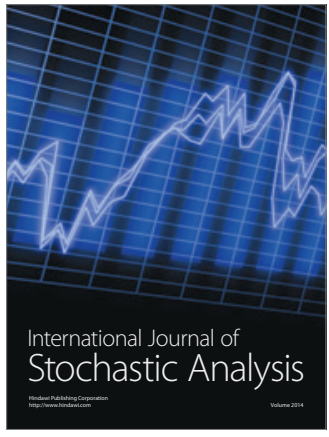

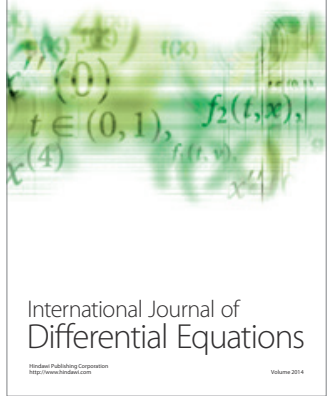
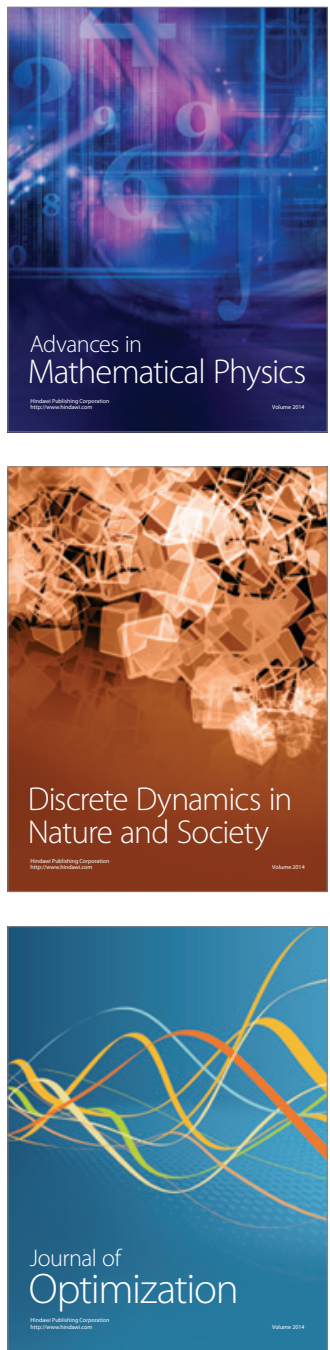